\title{
INFECTIOUS DISEASES, NSW: MAY 2000
}

\section{TRENDS}

Notifications of infectious diseases to the end of March 2000 were in line with seasonal expectations (Figure 2, Table 5).

\section{DRAFT PRIORITIES FOR COMMUNICABLE DISEASE CONTROL IN NSW, 2000}

The control of communicable diseases is a major function of any health system. Due to the multitude of diseases, and approaches to their control, health systems require a method for prioritising the development of new interventions to ensure efficient use of prevention resources.

\section{Communicable disease control structures in NSW}

In New South Wales, communicable disease prevention is primarily coordinated through the 17 Area Health Services' Public Health Units (PHUs). At a local level, PHUs work closely with other Area employees, primary and specialist health care providers, other government and non-government agencies, and the community to ensure that control programs are successfully implemented. At the state level, the NSW Department of Health's Health Protection Branch (including the Communicable Diseases Surveillance and Control, AIDSInfectious Diseases, Food and Environmental Health Units) develop policies in consultation with a range of advisory committees, and other agencies, based on available evidence.

\section{Control measures}

Communicable disease control and prevention require effective interventions in preventing human illness due to infection with micro-organisms or their toxins. The nature of such interventions varies widely according to the type of micro-organism or toxin involved. In general, interventions can be grouped into the following (overlapping) categories:

\section{Elimination of the causal micro-organism}

For example, cleaning, disinfecting or sterilising equipment, (such as surgical equipment, drinking water, toys in child care settings); cooking food; achieving world-wide immunity (such as smallpox, polio and measles vaccination); and the use of antibiotics (such as meningococcal disease, Haemophilus influenzae type b disease, pertussis, diphtheria, tuberculosis, and some sexually transmissible infections).

\section{Inhibition of the growth of the organism}

For example, refrigerating food; and the chemical treatment of water.

\section{Interruption of the transmission of the organism}

For example, hand washing; changing behaviours to maximise safe sex and minimise needle sharing; sterilisation of equipment; effective ventilation (to minimise the risk of respiratory infections); and the control of vectors (such as mosquitoes).

\section{Increase in host defences}

For example, immunisation, and better nutrition.

The effective implementation of such measures depends on a host of variables, including available resources, a committed and skilled workforce, community commitment, effective technology, collaboration and effective communication.

\section{Choosing priorities}

Public health priorities need to change with disease incidence, the implementation of effective programs, the cessation of programs, the emergence of new technologies, the emergence of new infections, and the changing incidence of known infections.

Recent developments have influenced the choice of prevention and control priorities, including the:

- 1998 Measles Control Program in which primary school children across Australia were immunised against measles, mumps and rubella. The unprecedented high level of immunity in the community now presents a new opportunity to focus on the elimination of measles and congenital rubella from Australia.

- recent development of a national strategy for hepatitis C surveillance

- emergence of multi-drug resistant tuberculosis (MDRTB) in many parts of the world

- emergence of antibiotic resistant pneumococcus in same parts of the world, and the development of effective vaccines against pneumococcal disease

- increasing recognition of nosocomial infections as a significant cause of morbidity

- persistent risk of congenital syphilis in some communities with reduced access to antenatal care

- the Olympic Games in Sydney in September 2000.

\section{Draft priorities}

The following list of priorities for communicable disease control facilitated by the NSW Department of Health is submitted for discussion:

- eliminate the transmission of measles

- eliminate congenital rubella 
- eliminate congenital syphilis

- better understand risk factors for new hepatitis C infections

- better understand risk factors for invasive pneumococcal disease

- better understand risk factors for nosocomial infections

- minimise the incidence of multi-drug resistant tuberculosis (MDR-TB)

- minimise the risk of communicable disease infections related to the Olympic Games in Sydney.

The identification of priority areas for communicable disease control will help focus long term strategies to achieve lasting health gains within existing resources. The following (largely existing) activities are required to achieve these goals.:

\section{Eliminate the transmission of measles}

- maximise MMR (measles-mumps-rubella) immunisation coverage among 12 month old and 4-5 year old children, through the Australian Childhood Immunisation Register, in collaboration with general practitioners (GPs), with the exclusion of cases and susceptible contacts from school and preschool;

- maximise MMR immunisation among all persons born after 1970;

- maximise MMR immunisation among overseas travellers, and persons working with overseas travellers;

- seek serological confirmation on all suspected cases of measles, and virological isolates from selected cases;

- rapidly implement control measures on the same day that a suspected case is reported to ensure that all contacts are immunised;

- investigate all reported cases of measles to identify failures of prevention and, therefore, implement better prevention strategies locally and state-wide.

\section{Eliminate congenital rubella}

- maximise MMR immunisation coverage among 12 month old and 4-5 year old children through ACIR, collaboration with GPs, exclusion of cases and susceptible contacts from school and preschool;

- ensure all women planning pregnancies are assessed for rubella immunity and are appropriately immunised;

- investigate all reported cases of congenital rubella to identify failures of prevention, and thereby implement better prevention strategies.

\section{Eliminate congenital syphilis}

- ensure that all populations at risk access appropriate antenatal screening and treatment for syphilis;

- identify all cases of congenital syphilis through laboratory and doctor-based surveillance;

- investigate all reported cases of congenital syphilis to identify failures of prevention, and thereby implement better prevention strategies, locally and state-wide.

\section{Monitor risk factors for new hepatitis $C$ infections}

- continue laboratory-based surveillance for hepatitis C antibodies;

- identify acute infections by writing to doctors managing persons with hepatitis $\mathrm{C}$;

- identify risk factors for infection among persons identified by their doctors to have acute infection;

- analyse and report on surveillance data on acute infections to assist in the development of better prevention strategies, locally and state-wide.

\section{Better understand risk factors for invasive pneumococcal disease}

Following advice from the NSW Infectious Disease Advisory Committee, in 2000 invasive pneumococcal disease will become notifiable by laboratories in NSW, in line with other Australian jurisdictions. Notification will allow analysis to determine the incidence of-and basic risk factors for-this condition that can be used to develop better prevention programs.

\section{Better understand risk factors for nosocomial infections}

The NSW Department of Health has funded a pilot project to examine the feasibility of nosocomial surveillance. A recent review of this project will aid in the development of better surveillance systems for hospital-acquired infections, and antibiotic resistant pathogens.

\section{Minimise the incidence and management of MDR-TB}

- ensure all suspected cases are promptly reported to public health units;

- ensure that all cases of TB receive supervised therapy to maximise compliance;

- ensure that all $M$ tuberculosis isolates are tested for drug sensitivities;

- ensure that the management of all persons with MDRTB is reviewed by NSW Health's Expert Panel.

\section{Minimise the risk of communicable disease infections related to the Olympic Games in Sydney}

The 2000 Sydney Olympics, with the influx of large numbers of persons from diverse parts of the globe, will present challenges for the surveillance and control of 
communicable disease outbreaks should they occur. The NSW Department of Health is working closely with other agencies to develop timely surveillance and control systems to minimise the risk of communicable disease transmission during this period.

\section{Maintaining existing programs}

Of course, the development of a list of priorities should not be at the expense of important existing programs. It is vital that we continue programs that include:

\section{Educate to prevent}

HIV infections, hepatitis $\mathrm{C}$, hepatitis $\mathrm{B}$, hospital infections, arboviral infections, and food borne illness.

\section{Needle \& syringe programs to prevent}

HIV infections, hepatitis C, and hepatitis B.

\section{Regulate to prevent}

Food borne illness, water borne illness, and legionnaires disease.

\section{Immunise to prevent}

Measles, mumps, rubella, Haemophilus influenzae type b disease, diphtheria, tetanus, pertussis, polio and hepatitis B (in everyone), and influenza, pneumococcal disease, Q fever, and yellow fever in those at risk.

\section{Intervene clinically to control}

Hepatitis A, meningococcal disease, Haemophilus influenzae type b disease, lyssavirus infections, tuberculosis, sexually transmissible infections, measles, pertussis, and hepatitis B.

\section{Maintain capacity to}

- detect, investigate and control outbreaks of disease

- monitor, investigate and prevent the spread of polio and diphtheria

- monitor, investigate and prevent spread of flaviviruses

- monitor, investigate and prevent the spread of exotic diseases

- monitor, investigate and prevent the spread of emerging pathogens

Comments on this draft list of communicable diseases control priorities are welcome, and should be made to Dr Jeremy McAnulty,

Communicable Diseases Surveillance and Control Unit, NSW Department of Health, Locked Mail Bag 961, North Sydney 2059; or by email at: jmcan@doh.health.nsw.gov.au.

\section{NSW PUBLIC HEALTH BULLETIN}

The NSW Public Health Bulletin is a publication of the NSW Department of Health. The acting editor is Ms Allison Salmon, Acting Manager, Public Health Training and Development Unit, NSW Department of Health. Dr Michael Giffin is production manager.

The Bulletin aims to provide its readers with population health data and information to motivate effective public health action.

\section{Submission of articles}

Articles, news and comments should be 1000 words or less in length and include a summary of the key points to be made in the first paragraph. References should be set out in the Vancouver style, described in the New England Journal of Medicine, 1997; 336: 309-315. Send submitted articles on paper and in electronic form, either on disc (Word for Windows is preferred), or by email. The article must be accompanied by a letter signed by all authors. Full instructions for authors are available on request from the editor.

\section{Editorial correspondence}

Please address all correspondence and potential contributions to The Editor, NSW Public Health Bulletin, Locked Mail Bag 961, North Sydney NSW 2059 or to Lmadd@doh.health.nsw.gov.au. Tel (02) 93919956 , Fax (02) 93919232.

\section{Distribution}

Please contact your local Public Health Unit or telephone (02) 93919942 to obtain copies of the NSW Public Health Bulletin or to notify us of a change of address. The Bulletin can be accessed via the Internet from the Department's Web site at: www.health.nsw.gov.au/public-health/phb/phb.html.

Back issues can be obtained from the Public Health Training and Development Unit, Locked Mail Bag 961, North Sydney NSW 2059. 


\section{FIGURE 2}

REPORTS OF SELECTED INFECTIOUS DISEASES, NSW, JANUARY 1995 TO MARCH 2000 , BY MONTH OF ONSET

These are preliminary data: case counts in recent months may increase because of reporting delays

\begin{tabular}{|cc|}
\hline \multicolumn{2}{|c|}{ NSW population } \\
Male & $50 \%$ \\
$<5$ yo & $7 \%$ \\
Rural & $42 \%$ \\
\hline
\end{tabular}

cases cases
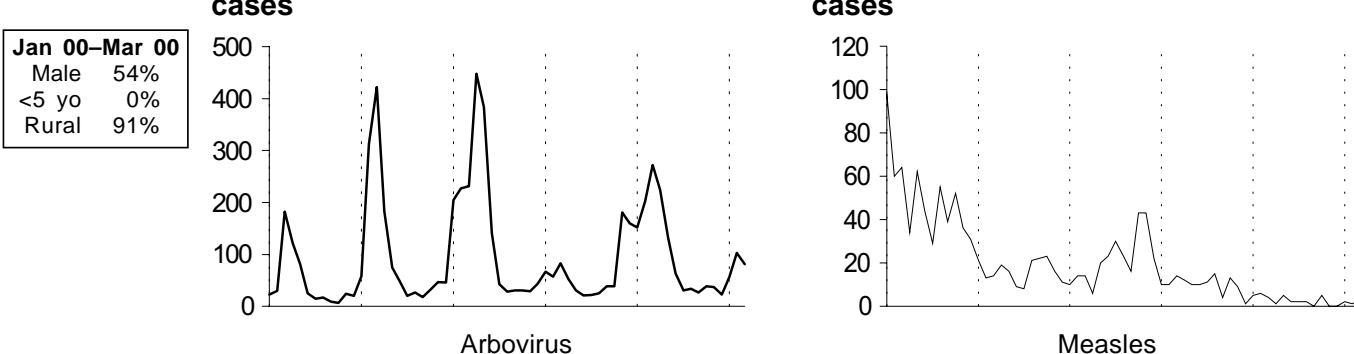

Jan 00-Mar 00

Male $33 \%$

$<5$ yo $33 \%$

$<5$ yo $0 \%$

Rural $67 \%$

Jan 00-Mar 00

500

\begin{tabular}{rr|r} 
Male & $68 \%$ & \\
$<5$ yo & $54 \%$ & 400
\end{tabular}

Rural $73 \%$

100

0

Cryptosporidiosis (not reportable before December 1996)

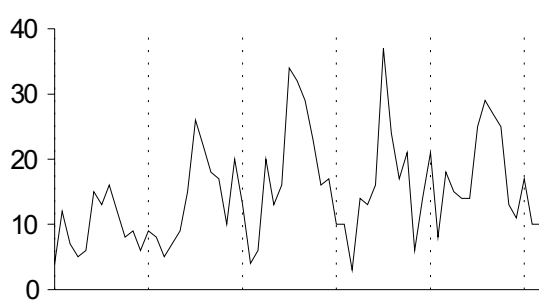

Jan 00-Mar 00

Male $62 \%$

$<5$ yo $19 \%$

Rural 43\%

\begin{tabular}{|cc|}
\hline \multicolumn{2}{|c|}{ Jan $\mathbf{0 0}-$ Mar $\mathbf{0 0}$} \\
Male & $92 \%$ \\
$<5$ yo & $0 \%$ \\
Rural & $18 \%$ \\
\hline
\end{tabular}
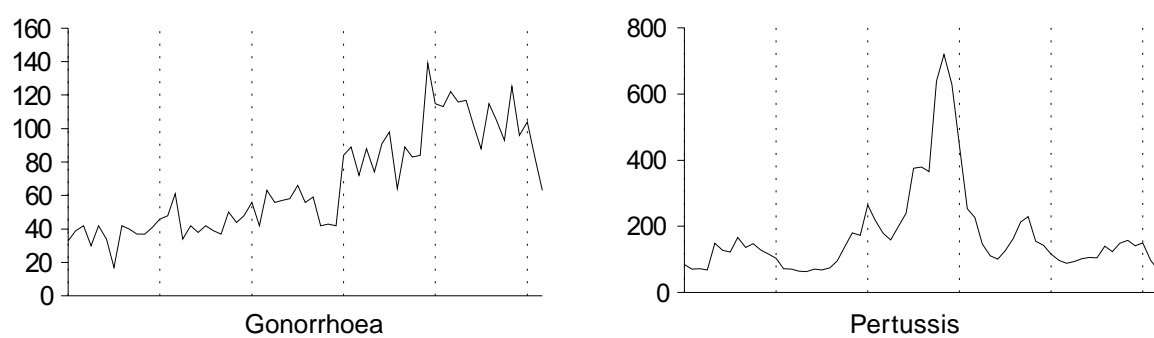

Jan 00-Mar 00

Male $42 \%$

$<5$ yo $12 \%$

Rural 59\%

Rural $18 \%$

Gonorrhoea

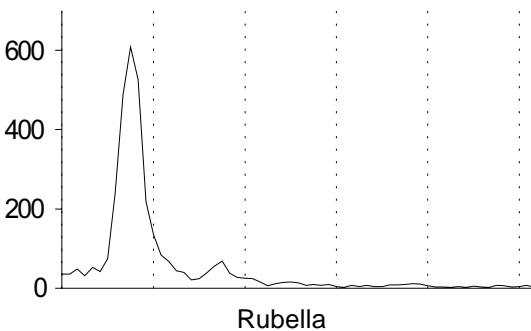

Jan 00-Mar 00

Male $77 \%$

$<5$ yo $\quad 0 \%$
Rural

Male $70 \%$

$<5$ yo $2 \%$

300

200

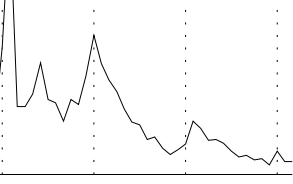

Hepatitis A

Rubella
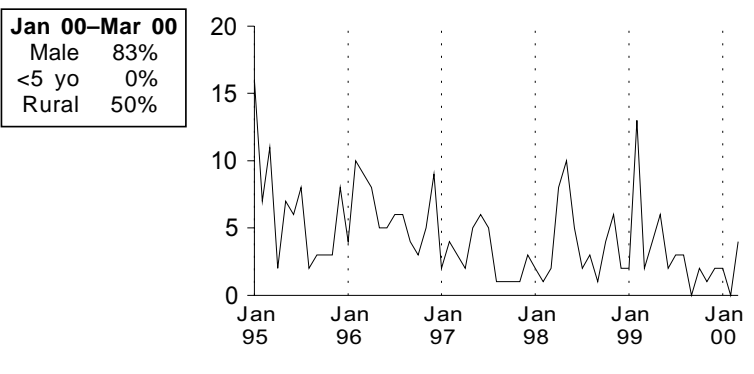

Legionella

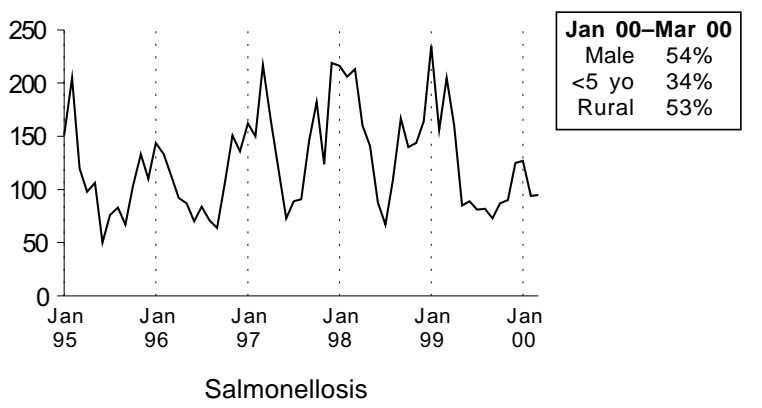


Blood-borne and sexually transmitted CSA NSA WSA WEN SWS CCA HUN ILL SES NRA MNC

NNC NEA MAC MWA FWA GMA

HIV infection*

Hepatitis B-acute viral*

Hepatitis B - other

Hepatitis C - acute viral

Hepatitis D - unspecified*

Hepatitis, acute viral (not otherwise specified)

Chancroid* $^{*}$

Chlamydia (genital) ${ }^{\star}$

Gonorrhoea

Syphilis

Vector-borne

Arboviral infection (BFV)*

Arboviral infection $(\mathrm{RRV})^{*}$

Arboviral infection (Other)

Malaria*

Zoonoses

Brucellosis*
Leptospirosis* $^{*}$

Q fever*

\section{Respiratory and other}

Blood lead level '

Legionnaires' Longbeachae*

Legionnaires' Pneumoph

Leprosy

Meningococcal infection (invasive)

Mycobacterial tuberculosis
Mycobacteria other than TB

Vaccine-preventable

Adverse event after immunisation

H.influenzae b infection (invasive)

Measles

Mumps*

Pertussis

Rubella*

Faecal-oral

Botulism

Cholera
Cryptosporidiosis
Giardiasis* $^{*}$

Food borne illness (not otherwise specified)

Gastroenteritis (in an institution)

Haemolytic uraemic syndrom

Hepatitis $A^{*}$
Hepatitis $E^{*}$

Listeriosis* ${ }^{*}$ Salmonellosis (not otherwise specified)

Salmonellosis (not otherwise
Typhoid and paratyphoid*

Typhoid and paratyphoid
Verotoxin producing Ecoli

* lab-confirmed cases only

CSA $=$ Central Sydney Area

$\mathrm{NSA}=$ Northern Sydney Area

SES = South Eastern Sydney Area NRA $=$ Northern Rivers Area $\mathrm{MNC}=$ North Coast Area
NEA $=$ New England Area $M A C=$ Macquarie Area

MWA $=$ Mid Western Area

$\mathrm{FWA}=$ Far West Area

GMA = Greater Murray Area $\mathrm{SA}=$ Southern Area 